\title{
A wonderful concert experience in Freiburg \ Konzert-ERLEBNISSE
}

\author{
Authors: Leander Hotaki \\ Submitted: 26. March 2019 \\ Published: $\quad$ 15. April 2019 \\ Volume: 6 \\ Issue: 4 \\ Affiliation: $\quad$ "Albert Konzerte" Freiburg, Germany. \\ Languages: \\ Keywords: \\ German \\ culture, music, concert, freiburg, Albert Konzerte \\ Categories: Performing Arts, Music \\ DOI: $\quad$ 10.17160/josha.6.4.548
}

\section{Abstract:}

Leander Hotaki is since 2010 the director of the German program called "Albert Konzerte" which is a program in charge of making possible to bring the greatest artist of the World to Freiburg and to make the most wonderful concerts in the south-west of Germany. In fact, he also promotes the work of the best students in the field of dramaturge or music in many universities in Germany and writes columns about different topics around music and musicians. This time he writes about the experience many people enjoy when they have the opportunity to interact with the artists and musicians from all over the world who perform in the concert hall in Freiburg. 


\section{Konzert-ERLEBNISSE}

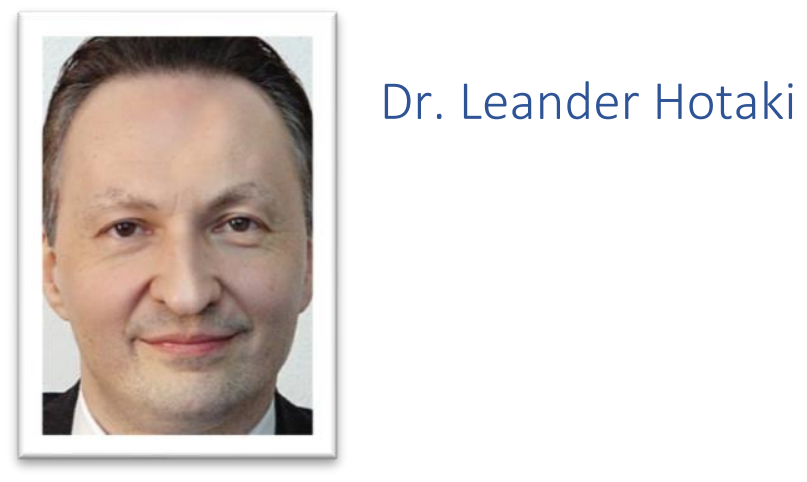

Es ist faszinierend, wie vielfältig das klassische Musikleben in Freiburg ist. Eine Stadt mit gerade einmal rund 220.000 Einwohnern und einer derart großen Anzahl von vorzüglichen Orchestern, Ensembles und Künstlern - können da die Auslastungszahlen der einzelnen Konzerte stimmen? Wer in Freiburg häufiger klassische Konzerte besucht, stellt fest, dass die meisten Konzerte sehr gut besucht sind. Noch immer gibt es hier ein Bildungsbürgertum, noch immer wird der Konzertbesuch mit Muse zelebriert. Natürlich, im Mittelpunkt steht für die meisten die Musik - darüber hinaus jedoch sind etliche Konzerte wie wichtige Opernpremieren oder das Albert-Konzert mit den Wiener Philharmonikern im April 2016, die viele gern mit einem Sektempfang und/oder einem guten Essen verbinden und gemeinsam mit dem Lebenspartner, der Familie, Freunden oder Geschäftspartnern erleben möchten.
Als Konzertveranstalter kann man diesen Bedürfnissen entgegenkommen, indem man die Konzerte im Einzelfall bereits um $18 \mathrm{Uhr}$ beginnt. So bleibt noch Zeit für einen schönen Ausklang des Konzertabends an einem Märzsonntag, auch wenn man zuvor noch längere Zeit in der Schlange der Autogrammjäger am CD-Verkaufstisch stand. Auch das scheint für eine zunehmende Zahl unserer Besucher wichtig zu sein im interaktiven Zeitalter: die Begegnung mit dem Künstler, das Bedürfnis, ihm seinen Dank auszudrücken, einen persönlichen Moment einzufangen, vielleicht ein gemeinsames Foto. Ein Treffen mit Freunden vor dem Konzert, ein künstlerisch begeisterndes Konzertprogramm, der Austausch in der Pause, persönliche Begegnung mit dem Künstler nach dem Konzert und dann noch ein Abendessen - so wird der Konzertbesuch zu einem doppelten Erlebnis: einerseits als gesellschaftliches Ereignis, andererseits als ganz persönliche, unverwechselbare und unersetzliche Erfahrung, die unvergessen bleibt. In einer Welt zunehmender Anonymisierung und digitaler Bespaßung kann man ein Publikum wie jenes in Freiburg, dass sich seine Konzerterlebnisse mit ungebrochener Intensität gönnt, nicht hoch genug schätzen. 